\title{
Unravelling the magic of latent safety threats
}

Yadin David

Forensic Engineering Section, Biomedical Engineering Consultants LLC, Houston, TX USA

\section{Correspondence to} Dr Yadin David, Forensic Engineering Section, Biomedical Engineering Consultants LLC, Houston, TX 77004-7071, USA; david@biomedeng.com

Accepted 13 October 2020 Published Online First 27 October 2020

\section{SLinked}

- http://dx.doi.org/10.1136/ bmjqs-2019-010740

\section{Check for updates}

(c) Author(s) (or their employer(s)) 2021. No commercial re-use. See rights and permissions. Published by BMJ.

To cite: David Y. BMJ Qual Saf 2021;30:265-267.
Sterile processing errors in medical and dental offices are ranked the third highest hazard according to the annual ECRI 'Top 10 Health Technology Hazards' 2020 report. ${ }^{1}$ Other experts have raised similar concerns with sterilisation processes. For example, the WHO and the Clinical Engineering Division of International Federation of Medical and Biological Engineering (IFMBE) have partnered to provide a series of webinars with international experts exchanging knowledge on COVID-19 related critical topics. A recent webinar addressed the critical challenge of decontamination and disinfection of COVID-19 medical equipment in low-income and middle-income countries. ${ }^{2}$ During the webinar, participants asked about methodologies to assess whether the transmission of infection is borne by technological tools used to fight the disease. How can critical lifesaving breathing equipment be safely and quickly sterilised and moved from one patient to the next? The WHO/IFMBE webinar ${ }^{2}$ stated that 'engineers and infection control professionals seem to be working in different silos'. Such silos must be dismantled because medical technology is indispensable in the provisioning of healthcare services. Disinfection and sterilisation of medical equipment are key concerns for healthcare organisations, and they require serious consideration of sociotechnical system interactions. The annual 'top 10 Health Technology Hazards report' is based on retrospective studies, yet management of COVID-19 safety requires capacity to process realtime data and the input of experts to predict where risks may occur and how to deploy plans to maintain a safe healthcare environment.

Alfred $\mathrm{et}_{\mathrm{al}} \mathrm{l}^{3}$ in this issue of BMJ Quality of Safety describe the Sterile Processing Department (SPD) as 'an example of a socio-technical system, where people, procedure, technology, environment, and organization interact to produce a range of proximal and distal outcomes'. ${ }^{3}$ The goal of their study was to 'develop a comprehensive understanding of the SPD assembly work system by uncovering key relationships between system components, and the sources of variance that might influence reliable assembly in instrument reprocessing'. They explain their findings as a function of a variety of contributing factors including: technological, labelling and human capacity issues. The authors' analysis cogently points to interventions 'beyond the hospital's traditional focus on individual staff'. Their results show the necessity of identifying system components and the impact of their interactions, to reveal appropriate interventions to improve the quality and safety of care and to reduce delays. The emphasis on expanding beyond 'tradition' is particularly pertinent now.

\section{SAFETY IS A DYNAMIC NON-EVENT}

The rapidly evolving COVID-19 epidemic has stretched the capacity of healthcare systems worldwide; consequently shining a light on existing quality and safety processes that often go unnoticed because, as Karl Weick advanced, safety is a dynamic non-event. ${ }^{45}$ Safety is dynamic because processes remain within acceptable limits due to moment-tomoment adjustments and compensations by the healthcare workers. It is a nonevent because safe outcomes are taken for granted and often go unrecognised. Paradoxically, the public appreciates quality and safety more when the system is unsafe, because lack of safety is salient, whereas normalcy is not.

You do not have to look far these days to see how much more appreciation the public has for the quality and safety of healthcare. However, this level of interest—concern, really-has the risk of 
isolating the responsibility for healthcare safety and quality to the (relatively) small group of professionals who actually deliver healthcare. It is further presumed to be their job to teach and apply medical, engineering and other scientific knowledge to improve the quality and safety of everything related to the healthcare services. However, we now realise that these presumptions need to be examined. Recent global conditions have demonstrated that national emergency response plans and the stocking of medical supplies fell short of expectations. Yet with the inclusion of experts specifically trained in safety and quality, these plans are already better networked with the supply chain that is being designed to be patient ready when urgently needed. Similarly, safety and clinical engineers were finally permitted to use ultraviolet radiation as sterilisation technology in patient care spaces. Ultraviolet radiation is not a new tool, but it is now applied because of expert recommendations and consequently it now plays an important role in protecting medical personnel, patients and family members. ${ }^{6}$

All of this begs the question: why has COVID-19 spurred recognition on the part of the public, and by medical professionals, of safety and quality controls in the delivery of healthcare? Pandemics highlight the existing people and processes that keep our systems safe and the fact that these elements of our system are not failsafe. There are threats in the system that remain latent because people are dynamically adapting in real time, but when the system is stretched too far, the threats no longer remain dormant. Deploying a predictive model of quality and safety, with professionals specifically trained in these areas, will be impactful to show in which scenarios such threats are likely to appear and can be timely mitigated.

\section{UNDERSTANDING WHAT KEEPS OUR SYSTEMS SAFE}

Without sufficient knowledge of what protects us, the ambiguous methodology for optimal provisioning of staff and patient safety was initially like performing magic. Starting with the elderly community and later with all ages, it has been like watching a Houdini act-how did that illusion which we were watching just happen? Will it happen again? As noted above, we need to re-examine the presumptions we hold about delivering safe and high-quality healthcare services. Once we understand the latent threats in the system, these can become controllable features. What appears to be magic is anything but. There are many system factors at play-people, processes and technologiesthat are keeping us safe, but they require further attention if they are to remain safe under unprecedented conditions.

Neither safety nor quality seems to have kept up in the rush to find an effective response to the pandemic. Whose job is it to keep up? As pointed out by Alfred et $a l^{3}$ regarding the instrument assembly process and a previous paper on the decontamination of instruments ${ }^{7}$ if we fail to identify all the system factors and their interactions, we fail to understand what keeps our systems safe. Consequently, we make assumptions about the backbones of our system, and when the system reaches its breaking point, we jump to solutions that are not aligned with the true root cause of the problem because we do not understand the mechanisms that underlie the safe operating state.

Previous examples regarding problems with instrument reprocessing have already pointed to the lack of understanding on the systems factors at play that led to poor solutions. For instance, I participated in an investigation in 2005 following the exposure of patients during surgeries in Duke University Health System, to instruments that were processed between procedures in hydraulic fluid instead of cleaning detergent. ${ }^{8}$ The hospitals did not detect the problem for weeks, despite complaints from staff members that the instruments felt unusually slick. The mix-up occurred when an elevator company drained hydraulic fluid into empty detergent barrels and the detergent supplier mistakenly redistributed them. The COVID-19 pandemic has emphasised the importance of decreasing instrument contamination risks ${ }^{9}$ as well as a general sense of urgency that may impose the risk of jumping to solutions too quickly. It is important to note that these latent safety threats have been there all along, but we are just noticing them more now than before because some of them are no longer latent. It was never magic; it was always the steady and adaptive coping of system factors-mostly people in the background-that never got recognised.

People around the world are now more clearly recognising their own responsibility and the benefits of adopting a more safety-oriented culture in their personal lives as well as in the products they use. The respect of paying homage to safety reached such a high awareness that one must again wonder, why did it take a global devastating pandemic to bring us to this level? And can the same be stated about quality?

\section{URGENT NEED TO IMPLEMENT APPROPRIATE SOLUTIONS FOR SOCIOTECHNICAL PROBLEMS}

Alfred $e \mathrm{al}^{3}$ in their analysis of sterile processing already pointed to the need 'for a wider range of interventions to enhance system performance beyond the hospital's traditional focus on individual staff behaviours and motivations'. System safety is thus dependent on the coordination of healthcare staff and management at the front line of service deliveries with the manufacturers who produce medical products, regulatory bodies and government who monitor its introduction into the market and clinical engineers who manage it over its life cycle use. This was acknowledged and highlighted by the 67th World Health Assembly when they issued a declaration in 2014 that states, in part, '[C]oncerned by the impact on patients of medical products of 
compromised quality, safety and efficacy, in terms of poisoning, inadequate or no treatment, contributions to drug resistance, the related economic burden, and erosion of public trust in the health system; ... urgent action is needed by the international community, Member States and relevant actors in health systems... to develop appropriate norms, standards and guidelines, including taking into account national, regional and international needs and initiatives,... to prioritize support for establishing and strengthening regional and subregional networks of regulatory authorities... to promote the greater participation of Member States in existing international and regional initiatives for collaboration and cooperation... to support the building-up of effective national and regional regulatory bodies and networks... to raise awareness of the importance of effective regulatory systems within the health system context'. ${ }^{10}$ In other words, system safety is dependent on coordination of all levels, not each level in isolation, and is a shared responsibility. Interventions to address disinfection and sterilisation risks are dependent on the coordination of all stakeholders, including the public.

As we begin to transform from fragility to vitality, this is the moment to convert the present heightened awareness into a strategy of education. Together, we should adopt guidelines for incorporating healthcare technology life cycle management beyond the focal point of products entry into the market and expand it to include consideration of the entire healthcare technology life cycles. From ideation to obsolescence, healthcare technology should be benchmarked at every stage based on indicators that every member including the public can understand, relate to and embrace as measure of minimum acceptable performance level for safety and quality. This will include the public, who for example may begin using home-based medical technology more than ever, in gaining participation to make care decisions. Every segment of care providers will now have tools to assess the whole life cycle of medical products from installation, performance assurance, upgrades, reallocation and retirement from one market to another.

Regardless of the reasons that led to relegating the quality and safety role only to those professionals who were formally tasked with it, we must embrace the strategy to expand the responsibility to the public. Instead of a single product mentality, let us challenge our ability to measure and embed predictive preventive measures of system performance. Critical characteristics of safety and quality management can be used to measure and mitigate latent risks and can be used to rank healthcare delivery and provide a 'report card' that can enhance choices the public can make. I suggest, therefore, a call for action to establish national institutions and international cooperation that will promote and harmonise safety and quality indicators relating to technological tools being deployed in our healthcare delivery systems.

No more magic show.

Funding The authors have not declared a specific grant for this research from any funding agency in the public, commercial or not-for-profit sectors.

Competing interests None declared.

Patient consent for publication Not required.

Provenance and peer review Commissioned; internally peer reviewed.

\section{REFERENCES}

1 Emergency Care Research Institute (ECRI). Top 10 health technology hazards for 2020. Expert insights from health devices. Special report, 2019. Available: https://www. ecri.org/landing-2020-top-ten-health-technology-hazards [Accessed Sep 27].

2 International Federation of Medical and Biological Engineering (IFMBE). Online course 7. Decontamination-Disinfection of critical COVID-19 equipment, health workers, and patients. Available: https://ced.ifmbe.org/covid19/guruPrograms/ 16-covid19/16-covid19-townhalls-decontamination.html [Accessed Sep 27].

3 Alfred M, Catchpole K, Huffer E. Work systems analysis of sterile processing: assembly. BMJ Qual Saf 2021;30:271-82.

4 Weick KE. Organizational culture as a source of high reliability. Calif Manage Rev 1987;29:112-27.

5 Reason J. Safety paradoxes and safety culture. Inj Control Saf Promot 2000;7:3-14.

6 Zhang J, Li J, Wu Z. The Chinese experience - fighting against COVID-19, Shanxi Medical engineers. Global Clinical Engineering Journal 2020;2:35-8.

7 Alfred M, Catchpole K, Huffer E, et al. Work systems analysis of sterile processing: decontamination. BMJ Qual Saf 2020;29:320-8.

8 The New York Times. Surgical Tools 'Washed' in Hydraulic Fluid. Available: https://www.nytimes.com/2005/06/13/us/ surgical-tools-washed-in-hydraulic-fluid.html

9 Aj B, Brown C, Abdelrahman T, et al. International surgical guidance for COVID-19: Validation using an international Delphi process - Cross-sectional study. Int J Surg 2020;79:309-16.

10 World Health Organisation. Regulatory system strengthening for medical products. Sixty-seventy World health assembly, 2014. Available: https://apps.who.int/gb/ebwha/pdf_files/ WHA67/A67_R20-en.pdf?ua=1 [Accessed Sep 27]. 